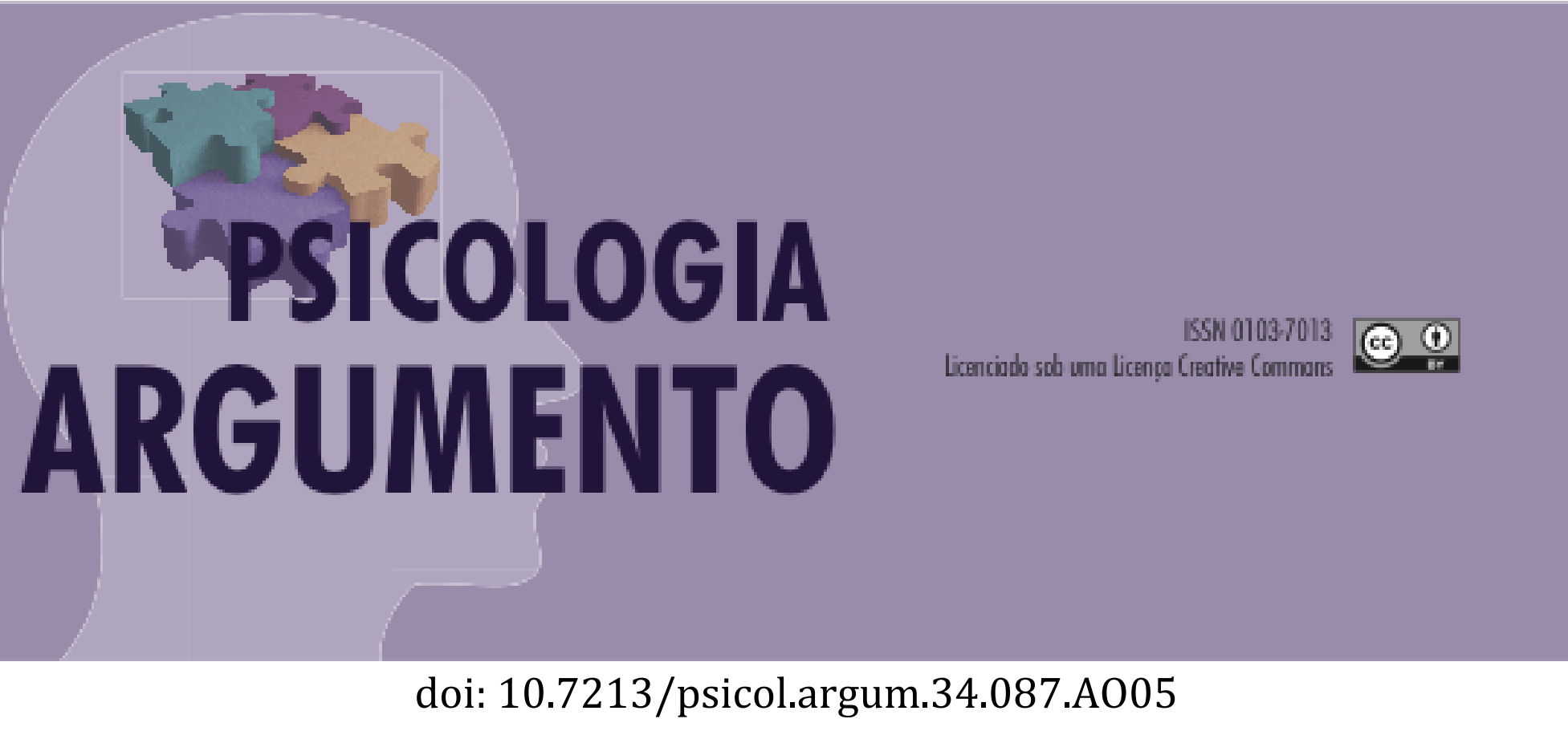

\title{
Condições psicológicas e comportamentos sexuais de adolescentes
}

\section{Psychological condition and sexual behavior of adolescents}

Nancy Ramacciotti de Oliveira-Monteiro[a], Rayssa Yussif Abou Nassif Menezes Ramos ${ }^{[b]}$

[a] Docente da Universidade Federal de São Paulo (UNIFESP); orientadora credenciada do Programa de Pósgraduação Interdisciplinar em Ciências da Saúde (UNIFESP), Santos, SP-Brasil,

e-mail: nancy.unifesp@gmail.com

[b] Pesquisadora do Laboratório de Psicologia Ambiental e Desenvolvimento Humano da Universidade Federal de São Paulo (UNIFESP), São Paulo, SP-Brasil, e-mail: rayssa.y.nassif@gmail.com

\section{Resumo}

$\mathrm{O}$ artigo apresenta um estudo sobre condições psicológicas e comportamentos sexuais de adolescentes em condição de vulnerabilidade social. Foram avaliados problemas psicológicos, competências e estados de identidade de 40 adolescentes, 12 a 18 anos, atendidos por um equipamento público de cidade da Baixada Santista (SP), voltado para desenvolvimento integral de jovens através de atividades socioculturais. 
Avaliação de comportamentos sexuais ocorreu com as meninas da amostra ( $\mathrm{N}=33)$. Os instrumentos utilizados foram o YSR (Youth Self-Report for Ages 11-18), o EOMEIS-II-II (Extended Objective Measure of the Ego Identity Status) e o QSJ (Questionário de percepções e práticas sobre comportamento sexual em jovens). Resultados indicaram tendências positivas na avaliação de competências e estados de identidade. Resultados de problemas psicológicos distribuíram-se em faixas não clínicas, limítrofes e clínicas. Quase metade das meninas referiu já ter tido relação sexual, início aos 15 e 16 anos, com indicativos de conhecimentos e práticas voltadas à contracepção. Os resultados apontaram para a importância de estratégias de apoio cultural para desenvolvimento positivo de adolescentes inseridos em contextos socialmente deficitários.

Palavras-chave: Adolescência. Vulnerabilidade. Competência. Identidade. Comportamentos sexuais.

\begin{abstract}
This article presents a study about psychological condition and sexual behavior of adolescents in a circumstance of social vulnerability. Were evaluated psychological problems, competences and states of identity of 40 adolescents, between 12 and 18 years of age, served by public equipment from a city in the region of Santos (SP), directed to the integral development of youngsters through social and cultural activities. The evaluation of the sexual behavior occurred with the girls in the sample $(N=33)$. The instruments used were YSR (Youth Self-Report for Ages 11-18), the EOMEIS-II-II (Extended Objective Measure of the Ego Identity Status) and the QSJ (questionnaire of perception and experience about sexual behavior in youngsters). The results indicated positive trends in the evaluation of competences and states of identity. Results indicating psychological problems were distributed in bands as non clinical, adjacent and clinical. Almost half the girls indicated having had sexual intercourse, beginning at 15 or 16, reporting previous knowledge of contraceptive practice. Results pointed to the importance of cultural support strategies for the positive development of adolescents inserted in contexts socially deficient.
\end{abstract}

Keywords: Adolescence. Vulnerability. Competence. Identity. Sexual development.

\title{
Introdução
}

Inserida como transição entre a infância e a idade adulta, a adolescência tem sido descrita como um dos marcos mais importante no processo de desenvolvimento humano. Por critérios da Organização Mundial da Saúde (OMS, 2014), essa etapa do desenvolvimento é delimitada no período compreendido entre os 10 e 20 anos de idade.

A passagem pela adolescência apresenta grande diversidade, por fatores individuais, sociais, culturais e históricos. As mudanças corporais advindas da puberdade são acompanhadas de crescimentos da ordem cognitiva, social e afetivo-emocional, em interação com elementos familiares e sociais. De forma geral, a adolescência é marcada por bons índices de saúde, vitalidade e criatividade (Assis, Pesce, \& Avanci, 2006), que são aspectos positivos do desenvolvimento. Por outro lado, autores de abordagem psicanalítica ressaltam diferentes conflitos e lutos próprios da adolescência (Sukiennik \& Salle, 2002) especialmente por perdas do corpo e da identidade infantil, e das relações com os pais, próprias da infância. A elaboração desses lutos levaria à afirmação do adolescente no mundo adulto.

Os produtos e processos do desenvolvimento do adolescente interagem com as condições sociais em que ele se insere. Por vezes, as condições de existência do adolescente 
podem ser tonalizadas por situações problemáticas, como no caso de vulnerabilidade social. A vulnerabilidade é identificada por Ruotti, Massa e Peres (2011) como a maneira pela qual os indivíduos enfrentam eventos adversos, sendo propensos a adquirirem certos comportamentos em resposta ao contexto. Esses eventos podem estar situados em dimensões sociais prejudicadas por pobreza, escassez de recursos escolares, esportivos, de lazer, de segurança e de locomoção.

Segundo a teoria ecológica do desenvolvimento (Barreto, 2016; Bronfenbrenner, 2001), o desenvolvimento humano relaciona-se com um conjunto de processos pelos quais as particularidades da pessoa e do ambiente interagem para alterações nas características da pessoa no curso de sua vida. Pelo desenvolvimento, há mudanças duradouras nas maneiras pela quais as pessoas percebem e lidam com seus ambientes. Por essa teoria ecológica, produtos do desenvolvimento emergem a partir de interconexões ambientais. Essas interconexões são estabelecidas em quatro níveis ambientais - os sistemas: micro, meso, exoe macro. $\mathrm{O}$ microssistema refere-se ao ambiente imediato, nas relações face a face estáveis, como na família. O mesossistema é um conjunto de microssistemas, e inclui interações com vizinhos, parentes, escola, ou projetos sociais, por exemplo. As interações com o exossistema acontecem sem que a pessoa em desenvolvimento esteja presente, como algumas interações com a rede de apoio social, e o trabalho dos pais. O macrossistema inclui os valores e crenças que são assimilados no desenvolvimento. Todos esses sistemas interagem com o desenvolvimento da pessoa.

As disfunções de desenvolvimento ocorreriam como manifestações recorrentes da dificuldade em manter o controle e a integração, através de situações presentes em diferentes domínios do desenvolvimento, como o biológico, o cognitivo, o social, e emocional, o artístico, entre outros. Em processos semelhantes, diferentes competências nos domínios do desenvolvimento vão emergir através das interações estabelecidas nesse contexto ambiental, composto pelos diferentes sistemas.

As competências humanas são diversificadas e aumentadas em complexidade nas diferentes etapas do ciclo vital, nesses diversos domínios do desenvolvimento. Também dessa maneira, o desenvolvimento da identidade ocorre durante toda a vida (Schwartz, Zamboanga, Meca, \& Ritchie, 2012).

A clássica contribuição psicossocial de Erikson (1997), voltada para características das diferentes etapas do ciclo vital, enfatiza a adolescência como fase mais importante para a constituição da identidade, na qual se define quem a pessoa é, o que quer ser, e com quais valores e metas o indivíduo estará solidamente comprometido. Segundo o autor, o desenvolvimento da identidade é crucial para a conversão do adolescente em adulto. A crise de identidade que ocorre na adolescência não terá no autor um sentido negativo, mas sim uma característica de encruzilhada em que alternativas diferentes são apresentadas para tomada de decisão. A formação da identidade recebe a influência de fatores intrapessoais (as capacidades inatas do indivíduo e as características adquiridas da personalidade), de fatores interpessoais (identificação com outras pessoas, incluindo família e grupos de iguais) e de fatores culturais (valores sociais em interação com os indivíduos).

A partir dessa proposta de Erikson, o psicólogo Marcia (1967) discorre sobre diferentes estados de identidade na adolescência, pelo interjogo de dimensões de exploração e de compromisso, em domínios interpessoais e ideológicos. 'Exploração' é a dimensão relacionada a exames de escolhas entre alternativas. A dimensão de 'compromisso' referese a escolhas relativamente firmes que são guias para futuras ações. Segundo Marcia, 
haverá quatro estados de identidade frutos da conjugação dessas duas dimensões. São elas: a difusão, o pré-fechamento, a moratória e a identidade estabelecida (Marcia, 1967; Schoen-Ferreira, 2007).

No estado de difusão, o indivíduo não exercita explorações e também não apresenta compromissos ou decisões, não se sentindo internamente pressionado para tanto. A difusão pode representar um estado inicial do desenvolvimento da identidade, característico dos primeiros anos da adolescência. Os indivíduos que permanecem nesse estado terão dificuldades com os papéis sociais e com os próprios sentimentos. Para Kimmel e Weiner (1998), por exemplo, quando o adolescente persiste no estado de difusão, ele tenderá a desenvolver transtornos psicológicos.

No estado de pré- fechamento, o indivíduo se compromete com alternativas sem ter tido explorações adequadas; suas metas ideológicas são eleitas por outros, e a pessoa adota crenças, por exemplo, sem fazer críticas a respeito. Nesse estado de identidade, não ocorrem experiências de exploração e o comprometimento é, em geral, definido pelos pais e pela cultura. Para Marcia (1967), o estado de pré-fechamento pode estar relacionado com o autoritarismo, tanto dos pais quanto do próprio adolescente. Esse também é um estado mais encontrado nos anos iniciais da adolescência.

Já no estado de moratória, tido como o mais característico da adolescência, conforme Erikson (1997), o indivíduo depara-se com diversos temas a respeito da sua identidade, sendo que ainda não definiu suas escolhas. Nesse sentido, ele estaria explorando alternativas ativamente, porém sem estabelecer compromissos. A moratória é o estado precursor da identidade bem estabelecida, tratando-se de um período de transição entre a segurança da infância e a autonomia do adulto. Na moratória, o adolescente explora opções e experimenta diferentes papéis e modos de agir, para que assim possa descobrir como se comportar de forma a ter sentido para si mesmo e estabelecer a sua identidade.

Por fim, o estado de identidade estabelecida caracteriza-se pela obtenção, conquista e construção da identidade, já que o indivíduo fará escolhas após ter passado por um período de experimentação. Esse estado é mais esperado nos anos finais da adolescência e início da terceira década de vida.

Conforme Matteson (1972), na adolescência não ocorre uma única crise de identidade. Os jovens vão se confrontando com diversas alternativas durante esse período do desenvolvimento. No principio da adolescência, ocorrem mais explorações associadas às mudanças corporais; no final da adolescência, emergem maiores confrontos com as ideologias. Os estados de identidade esperados para o início da adolescência, o de difusão e o de pré-fechamento, são menos evoluídos ou 'passivos'. Já a moratória e a identidade estabelecida são estados de identidade mais evoluídos ou 'ativos', e são esperados para uma etapa mais avançada da adolescência (Zacarés, Iborra, Tomás, \& Serra, 2009).

De acordo com Kimmel e Weiner (1998), quanto mais desenvolvido o sentimento de identidade, mais o sujeito valoriza o modo em que é parecido, ou diferente, dos demais e mais claramente reconhece suas limitações e habilidades. Quanto menos desenvolvida está a identidade, mais é necessário o apoio de opiniões externas para a auto-avaliação e para compreender as pessoas como distintas.

Como qualquer domínio do desenvolvimento humano, o desenvolvimento da identidade emerge das constantes interações entre as condições ambientais e características pessoais (Tesouro, Palomanes, Bonachera, \& Marínez, 2013). Da mesma forma, ocorre o desenvolvimento de padrões de comportamentos sexuais, especialmente aqueles mais 
relacionados à procriação e formação de famílias. Os comportamentos sexuais estão inseridos em um conjunto complexo de referências pessoais, ambientais, e cronológicas (tempos históricos e etapas temporais do desenvolvimento humano).

Na sociedade urbana ocidental contemporânea, os adolescentes têm amplo contato com referências do grande valor do sexo e da liberdade sexual, através da mídia e das redes sociais. Concomitantemente, existem expectativas sociais de que os jovens sejam autônomos e responsáveis quanto à prática de sexo seguro, como no uso de métodos preventivos para evitar doenças sexualmente transmissíveis (DSTs) e também para a contracepção (Reis \& Oliveira-Monteiro, 2007).

Desde a década de 1980, com a epidemia da AIDS e o número crescente de gestações durante a adolescência, houve uma maior visibilidade para a sexualidade juvenil, aumentando a orientação e o uso de preservativos. Apesar dessa melhora, o uso adequado de métodos anticoncepcionais nem sempre é frequente (Teixeira, Knauth, Jandyra, \& Leal, 2006). Outro aspecto relevante da sexualidade dos adolescentes é aquele referente a questões de gênero, principalmente no tocante à idade e ao parceiro da primeira relação sexual. Na maioria das vezes, a iniciação sexual de jovens do sexo masculino ocorre mais precocemente que a de jovens do sexo feminino (Ministério da Saúde, 2008).

Dias e Gomes (1999) comentaram que nos diálogos entre adolescentes e suas famílias, dificilmente se encontravam aberturas necessárias para a discussão relativa à sexualidade. Muitos jovens sentem-se constrangidos ou temem a desaprovação de seus pais, enquanto vários pais sentem-se despreparados para abordarem o assunto. É frequente que a sexualidade da filha só seja discutida quando ela dá sinais mais evidentes de início de vida genital ativa ou engravida; essa dificuldade na aceitação da sexualidade da jovem restringe a ocorrência de diálogos eficientes sobre métodos contraceptivos.

Embora as taxas de fecundidade de adolescentes venham diminuindo no Brasil, em territórios de periferias sociais e urbanas, o fenômeno da gravidez na adolescência ainda é recorrente (Bernardo \& Oliveira-Monteiro, 2015). Projetos de natureza cultural, artística, e esportiva têm sido inseridos nesses territórios de vulnerabilidade social em busca da promoção da saúde integral de crianças e adolescentes. $\mathrm{O}$ escopo de alguns desses projetos abarca medidas de prevenção relacionadas à temática da sexualidade. Tais projetos podem oferecer oportunidades aos adolescentes para encontro de perspectivas de vida, baseadas em trocas interpessoais positivas, elaboração de valores, sentido de pertinência pessoal e social, e possibilidade de trabalho (Prado, 2008). Em investigação sobre comportamentos sexuais de adolescentes em situação de vulnerabilidade social, inseridos em projeto cultural, Reis e Oliveira-Monteiro (2007) encontraram dados de que aqueles adolescentes não recebiam informações sobre sexualidade em fontes familiares e manifestavam que a idade melhor para ter um primeiro filho seria na vida adulta.

Em concordância com o exposto, os elementos psicossociais do desenvolvimento incluindo competências sociais, condições emocionais e comportamentais positivas, estados de identidade desenvolvidos a partir de explorações realizadas na adolescência, e comportamentos sexuais associados à saúde integral - seriam dimensões emergentes de interações de condições pessoais com elementos dos ecossistemas (micro, meso, exo, e macro). Considerando que adolescentes em condições de vulnerabilidade social possuem, por definição, menor probabilidade de interagir com bons recursos sociais pertinentes a todos esses sistemas, eles teriam maiores déficits na emergência de aspectos psicossociais positivos do desenvolvimento? 
O estudo aqui apresentando partiu dessa indagação propondo como objetivo explorar indicadores de condições psicossociais de um grupo de adolescentes em situação de vulnerabilidade social que frequentavam um equipamento público de atendimento sociocultural. Foram verificados competências, problemas psicológicos, estados de identidade, e comportamentos sexuais - dimensões do desenvolvimento humano presentes em seu domínio psicossocial.

\section{Método}

\section{Participantes}

Participaram da pesquisa 40 adolescentes, 33 meninas e sete meninos, idades de 12 a 18 anos, usuários de um equipamento social público de uma cidade de médio porte da Baixada Santista, região metropolitana do Estado de São Paulo. Nesse equipamento eram oferecidas atividades socioculturais para jovens moradores da região onde estava instalado. Essas atividades incluíam oficinas sobre temas voltados à adolescência (sexualidade, escolaridade, sociabilidade, empregabilidade) e informática, e aulas de arte (como desenho e teatro).

Os jovens atendidos no projeto eram considerados em condições de vulnerabilidade social pela pertinência àquele território de periferia urbana e social, permeado por pobreza, criminalidade, uso abusivo de drogas, e poucas opções de esporte e lazer. A amostra foi constituída pelo critério de intencionalidade e conveniência, não se caracterizando como probabilística.

Foram convidados à participação na pesquisa 50 adolescentes que frequentavam esse projeto sociocultural. Todos manifestaram interesse na pesquisa, mas 10 não puderam participar porque não foi obtida a autorização de seus responsáveis. Esses responsáveis indicaram verbalmente (via pessoal e por telefone) ressalvas quanto à aplicação de questionário sobre comportamentos sexuais (o que estava informado no Termo de Consentimento Livre e Esclarecido apresentado).

\section{Instrumentos}

No levantamento de dados foram utilizados os seguintes instrumentos:

Youth Self-Report for Ages 11-18 (YSR): o Inventário de Auto-avaliação para Adolescentes de 11 a 18 anos compõe a Bateria de Sistema de Avaliação Empiricamente Baseado de Achenbach (ASEBA) de Achenbach e Rescorla (2011). Os autores do instrumento não o consideram na categoria de teste psicológico, mas si, como um instrumento de triagem que pode ser utilizado por membros de equipes de saúde. O inventário consiste num questionário que identifica, sob a ótica dos adolescentes, condições em duas escalas: a) 'escala de competências', que inclui as sub-escalas de 'competência total', 'competência social' e 'atividades', avaliando condições de amizades, participação em grupos, tarefas realizadas habitualmente, esportes e passatempos; e b) 'escala de problemas de psicológicos', nas sub-escalas de problemas internalizantes/emocionais (que incluem ansiedade/depressão, isolamento/depressão e queixas somáticas) e problemas externalizantes (que incluem comportamentos de quebra de regras e comportamentos agressivos). Os dados do YSR foram tratados pelo software de correção do inventário (Assessment Data Manager 
Program - ADM). Os escores obtidos são classificados nas faixas: 'não clínica' (indicativa de condições positivas), 'limítrofe' ou 'clínica' (indicativas de preocupações e necessidade de intervenção). Para a escala de competências, escores abaixo de 37 são considerados clínicos e acima de 40, não clínicos; escores intermediários (37 a 40) referem-se à faixa limítrofe. Para os problemas psicológicos, escores abaixo de 64 são da faixa 'não clínica', entre 64 e 70 da faixa 'limítrofe', e, acima de 70 da faixa 'clínica'. O YSR é um dos inventários mais citados na literatura internacional para avaliação de problemas psicológicos de competência em adolescentes. O instrumento foi validado no Brasil por Rocha (2012).

Extended Objective Measure of the Ego Identity Status (EOMEIS-II): um instrumento de estudo sobre identidade com 64 afirmações que abrangem os domínios: 'ideológico' e 'interpessoal'. Cada uma das afirmações reflete características de um dos quatro estados de identidade: 16 fazem referência ao estado de identidade estabelecida; 16 ao estado de moratória; 16 ao estado de difusão; e outras 16 ao estado de pré-fechamento. Os adolescentes valoram cada item em uma escala tipo Likert de seis pontos, desde 'discordo totalmente' até 'concordo totalmente'. Os domínios 'ideológico' e 'interpessoal' contemplam quatro áreas, com oito afirmações para cada área: a) áreas da escala do domínio ideológico (I- ocupacional; II- religião ou crenças religiosas; III- política; e IV- filosofia de vida); b) áreas da escala do domínio interpessoal (I- lazer ou tempo livre; II- amizade; III- papel de gênero; e IV- relacionamento amoroso). A somatória das respostas assinaladas pelo individuo permite ao pesquisador traçar o estado de identidade em cada um dos domínios em que o investigado estava no momento em que respondeu à escala. A validação semântica do instrumento para a língua portuguesa foi conduzida por Schoen-Ferreira, Meneghuelli e Aznar-Farias (2005).

Questionário de percepções e práticas sobre comportamento sexual em jovens (QSJ): esse questionário propõe investigar dados sobre aspectos de vida sexual de jovens, com ênfase em temas relacionados à procriação. A formulação de várias perguntas do questionário foi baseada em elementos de conteúdo e linguagem do próprio universo de percepções e narrativas de adolescentes pesquisadas em estudos sobre gravidez e maternidade de adolescentes em situação de vulnerabilidade social (Oliveira-Monteiro, Freitas, \& Aznar-Farias, 2014; Oliveira-Monteiro, Nascimento, Montesano, \& Aznar-Farias, 2013).

\section{Procedimentos}

O projeto teve aprovação de Comitê de Ética em Pesquisa (CEP/UNIFESP $n^{\circ}$ 121009). A partir do contato prévio com a instituição pública que abrigava o projeto sociocultural, foram realizados convites para a participação, para os adolescentes e seus responsáveis. Com a concordância e aceite, foram lidos e assinados Termos de Consentimento Livre e Esclarecido (TCLE) para os responsáveis, e Termos de Assentimento para os adolescentes participantes.

Para finalizar o levantamento de dados foram necessárias 24 idas ao campo (local onde funcionava o equipamento). A presença dos adolescentes usuários do projeto era inconstante e imprevisível. A aplicação dos instrumentos acontecia num mesmo dia, em horários combinados com a equipe gestora do projeto, os demais profissionais, e os próprios adolescentes, em local com privacidade dentro do próprio equipamento. Essa aplicação tinha duração aproximada de uma hora para cada um dos participantes. 
Os instrumentos eram autoaplicáveis, porém alguns adolescentes mostraram dificuldades de entendimento de questões do YSR, sendo-lhes fornecidos esclarecimentos. Apesar de instruções iniciais com explicações a respeito, alguns adolescentes também manifestaram dificuldades para pontuarem a escala do tipo Likert do EOMEIS-II. Durante a aplicação do QSJ, alguns adolescentes pareciam inibidos e retomavam a discussão sobre o sigilo da pesquisa, informado na leitura do Termo de Assentimento.

Para obtenção dos resultados, dados do YSR foram tratados pelo software ADM (Assessment Data Manager Program) de correção do inventário. Os resultados do EOMEIS-II foram obtidos pela soma referente a cada um dos domínios de estados de identidade. Embora o instrumento QSJ tenha sido aplicado em toda a amostra $(\mathrm{N}=40)$, somente os questionários das meninas $(\mathrm{N}=33)$ foram analisados, para elementos descritivos de frequência de resposta, visto o número muito pequeno de meninos na amostra.

\section{Resultados}

\section{Resultados do YSR}

Na Figura 1 são apresentados resultados do YSR nas três escalas de Competência (competência total, social e atividades), de meninas e meninos da amostra.
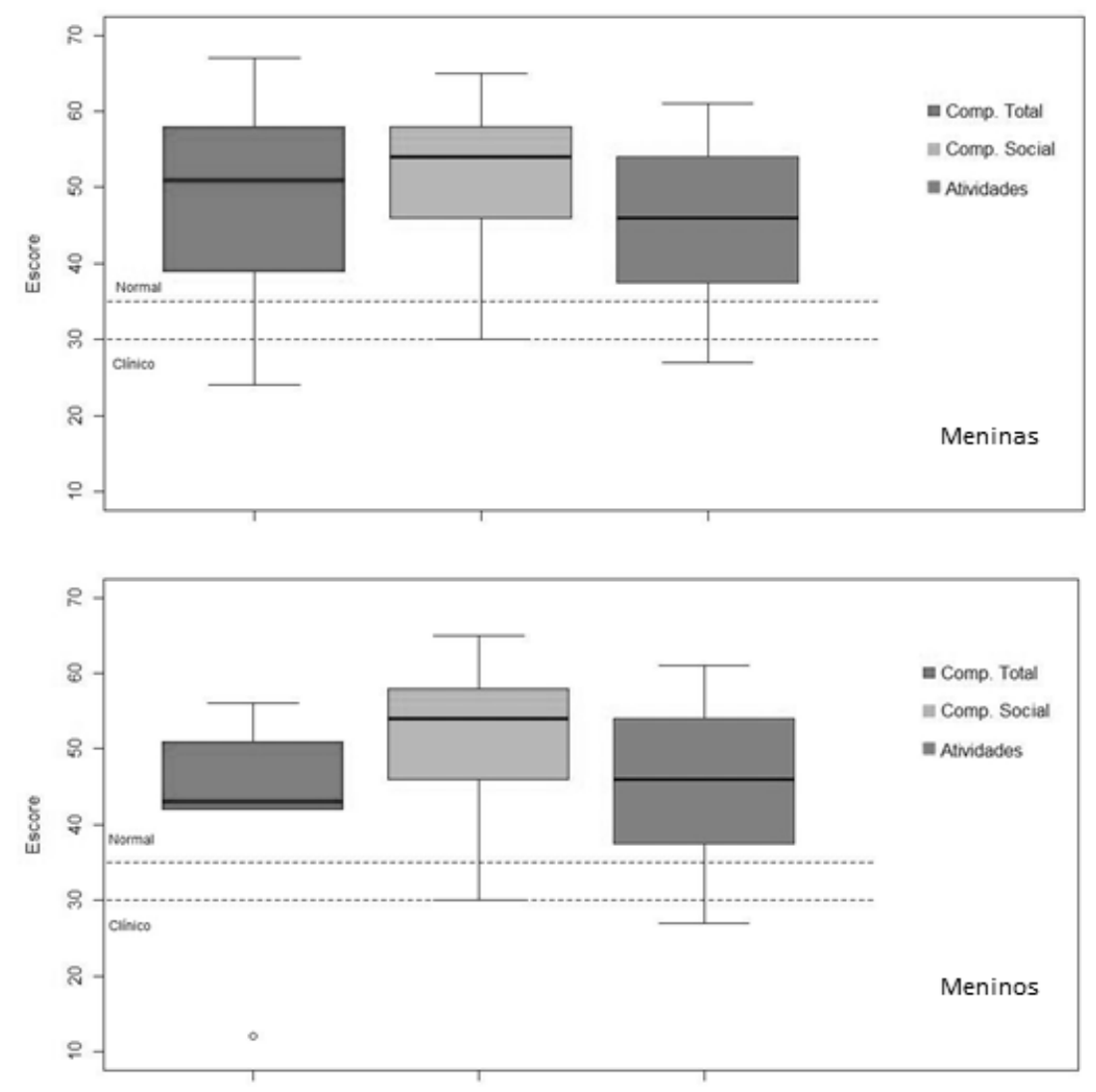

Figura 1. Distribuição de dados sobre Competências em meninas e meninos (YSR)

Conforme dados da Figura 1, as meninas e os meninos da amostra apresentaram resultados na faixa não clínica, em todas as sub-escalas de Competência avaliadas pelo YSR. 
A Figura 2 refere-se aos resultados do YSR quanto a Problemas internalizantes (emocionais) e externalizantes (comportamentais), para meninas e meninos da amostra, respectivamente.
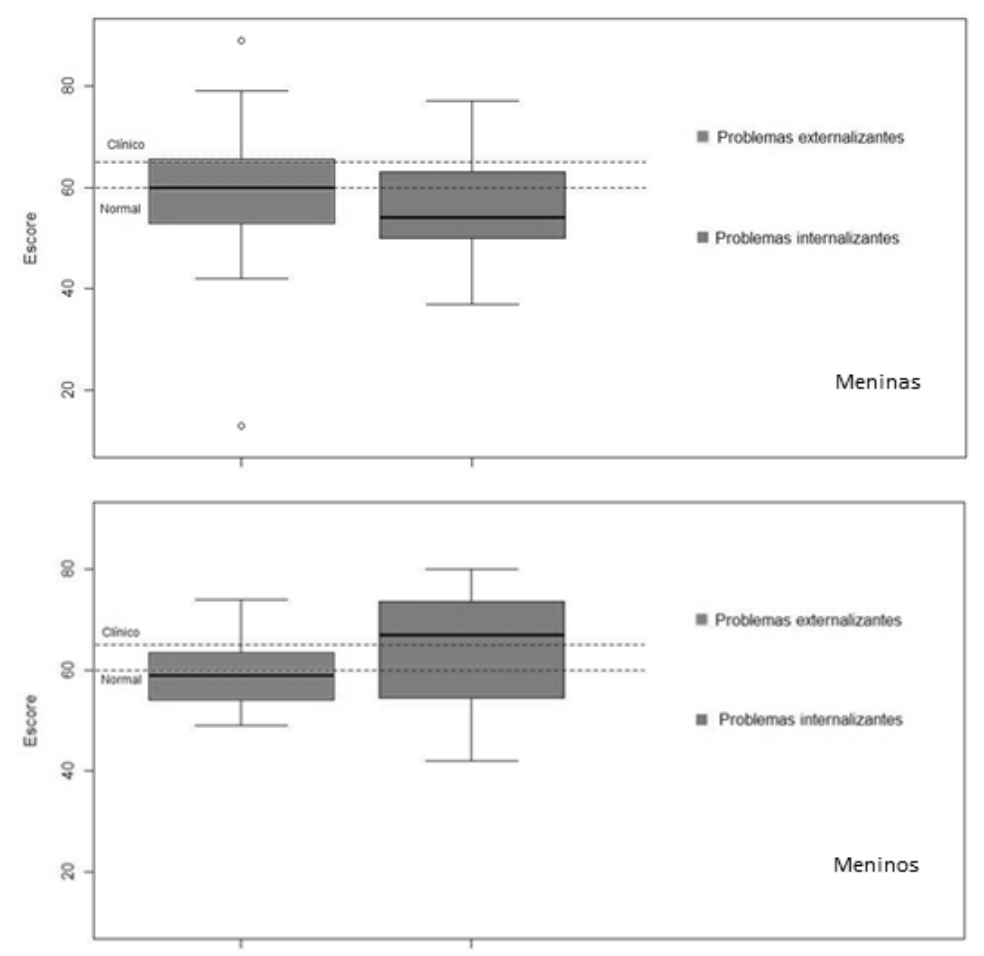

Figura 2. Distribuição de dados sobre Problemas internalizantes e externalizantes

Como indicado na Figura 2, as meninas da amostra tiveram resultados distribuídos nas sub-faixas não clínica e limítrofe, tanto para problemas externalizantes (comportamentais) como para problemas internalizantes (emocionais). Já os meninos tiveram resultados distribuídos para as faixas não clínica e limítrofe, em problemas externalizantes. Os resultados relativos a problemas internalizantes, nos meninos, atingiram a faixa clínica, além das faixas não clínica e limítrofe, em sua distribuição.

\section{Resultados do EOMEIS-II}

As Figuras 3 e 4 apresentam resultados percentuais do EOMEIS-II, relativos a estados de identidade da amostra, nas sub-faixas etárias de adolescentes mais novos (12 a 15 anos) e mais velhos (16 a 18 anos). Como mostrado nessas figuras, o estado de difusão foi o predominante nos adolescentes mais novos da amostra, nos dois domínios (ideológico e interpessoal). Nos adolescentes mais velhos, o estado de moratória foi o mais indicado, também nos dois domínios. 


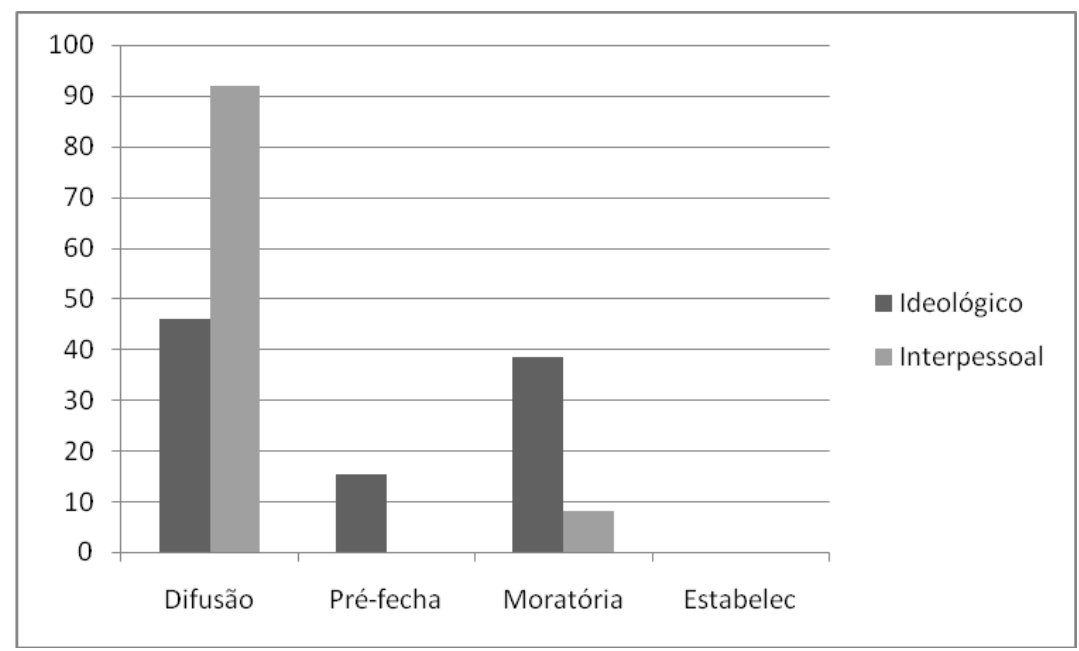

Figura 3. Dados percentuais dos estados de identidade do sub-grupo de 12 a 15 anos (EOMEIS-II)

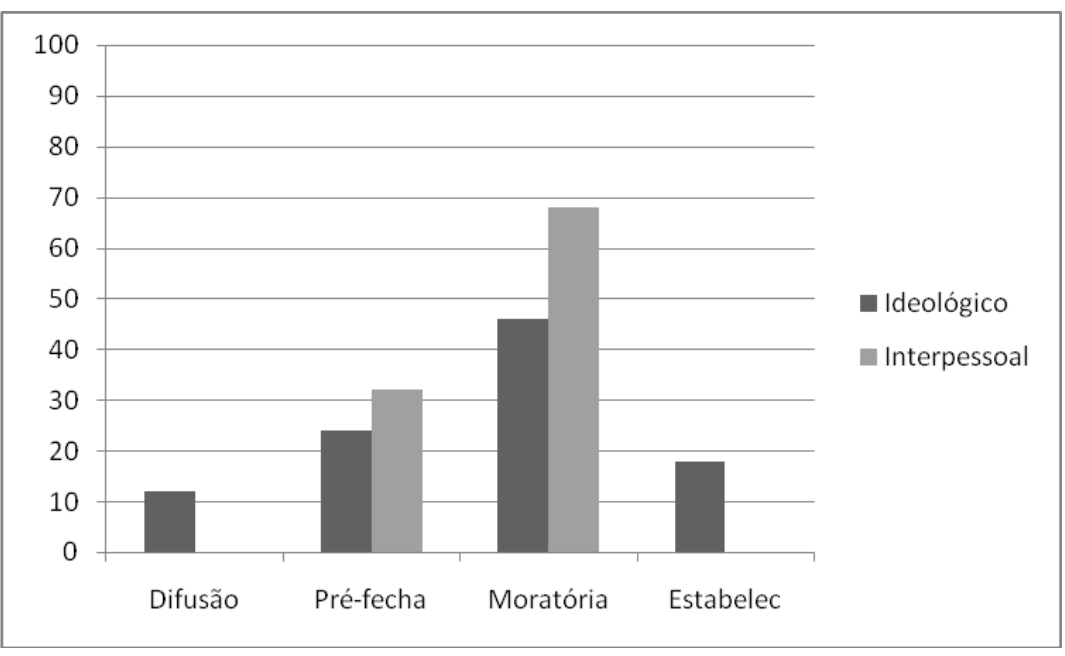

Figura 4. Dados percentuais dos estados de identidade do sub-grupo de 15 a 18 anos (EOMEIS-II)

\section{Resultados do QSJ}

Os resultados do QSJ indicaram que, para as adolescentes investigadas, as fontes de informação sobre sexualidade vieram principalmente da escola (70\%) e da mídia (37\%). As adolescentes podiam indicar diferentes fontes, de forma não exclusiva. A porcentagem das adolescentes que referiram já terem tido relação sexual foi igual à das que referiram não ter tido (45,5\%); $9 \%$ das meninas não responderam à indagação a respeito. Ainda quanto ao tema da primeira relação sexual da amostra investigada, 35\% afirmaram ter tido a primeira relação sexual antes dos 15 anos. A referência da primeira relação aos 16 anos apareceu em $41 \%$ dessas meninas.

Com relação a métodos contraceptivos, daquelas meninas que afirmaram já terem tido relação sexual, $87 \%$ referiram uso de preservativo, 33\% de contraceptivo oral (pílula) e 6\% de injeção. O instrumento também investigou qual seria a idade que a adolescente julgava melhor para ter o primeiro filho. Nenhuma das adolescentes da amostra indicou 
que julgava a melhor idade para ter um primeiro filho dentro da faixa da adolescência; todas deram indicadores de idades superiores à faixa de 20 anos, sendo que $18 \%$ referiu idades superiores a 30 anos. Quando indagadas sobre os motivos que levariam uma adolescente a engravidar, não houve referências de dificuldades para comprar contraceptivos; $70 \%$ das adolescentes investigadas afirmaram que esse não era um motivo para a ocorrência da gravidez.

\section{Discussão}

Partindo da concepção de Bronfenbrenner (2001) de que o desenvolvimento humano emerge de interações com diferentes sistemas ambientais, desde os mais proximais até aqueles em que o indivíduo não está presente, o desenvolvimento positivo de adolescentes em situação de vulnerabilidade social, como os estudados nesta pesquisa, necessitariam de intervenções para incremento de recursos evolutivos, também de natureza psicossocial dimensão específica em que se encontra a vulnerabilidade dessa população. Este estudo procurou verificar, num pequeno grupo de adolescentes nessas condições de vulnerabilidade, mas que passavam por experiências oferecidas por um equipamento de apoio psicossocial, algumas dimensões de seu desenvolvimento. Foram avaliados competências, problemas psicológicos, estados de identidade e comportamentos sexuais desses jovens.

De forma geral, tendências positivas desses aspectos de desenvolvimento foram encontradas nas avaliações, quanto aos indicativos de competências e de estados de identidade. Oportunidades de exploração de elementos culturais e artísticos diversificados, para além dos presentes no microssistema familiar e nos mesossistemas (Bronfenbrenner, 2001) permeados por condições de desenvolvimento da identidade (prevenção frente a compromissos estabelecidos precocemente).

As competências avaliadas (de ordem social e de atividades, abrangendo amizades, participação em grupos, tarefas realizadas habitualmente, esportes e passatempos), indicaram graus positivos em toda a amostra. Da mesma forma, os estados de identidade menos desenvolvidos e passivos (difusão e pré-fechamento) (Zacarés et al., 2009) foram encontrados especialmente nos adolescentes mais jovens, condição esperada no desenvolvimento da identidade (Marcia, 1967). Nos adolescentes mais velhos, esses estados mais imaturos foram menos presentes, e o estado de moratória, mais ativo e maduro (Zacarés et al., 2009) foi o mais característico nesse subgrupo. Para Erikson (1997) a moratória é característica da adolescência permitindo a evolução para o estabelecimento da identidade.

Já quanto a problemas psicológicos de natureza externalizante (comportamentais), os resultados se distribuíram nas faixas não clínica e limítrofe (essa última indicativa de algumas preocupações de ordem clínica). Resultados de problemas de natureza internalizante (emocionais) também se distribuíram nas faixas não clínica e limítrofe, chegando a atingir a faixa clínica, nos meninos investigados, indicativos de dificuldades psicológicas nesses adolescentes meninos (Sandhu, 2015). Os problemas emocionais avaliados pelo YSR abarcam dificuldades nos âmbitos de ansiedade e depressão e esse resultado indicou que alguns meninos da amostra demandavam intervenções psicológicas. Essa verificação aponta para a possibilidade de que aspectos emocionais não sejam tão permeáveis às intervenções psicossociais.

Finalmente, os resultados relativos a comportamentos sexuais, segundo referência das meninas pesquisadas, mostraram indicativos da possibilidade de conhecimentos e 
práticas seguras frente à gravidez na adolescência, condição que também pode ser associada às intervenções nas oficinas de sexualidade oferecidas pelo equipamento em que elas estavam inseridas.

Para a maioria das meninas, a escola e a mídia haviam sido a principal fonte de informações sobre assuntos de sexualidade. Como em Reis e Oliveira-Monteiro (2007), as adolescentes manifestaram que não recebiam orientações a partir da família. A dificuldade das famílias das adolescentes para lidarem com assuntos pertinentes à sexualidade com seus filhos, como também citada por Dias e Gomes (1999), também ficou indicada pela manifestação de recusa de consentimento, para um quinto dos convites para a participação na pesquisa, com alegação de motivo a partir da aplicação de questionário sobre comportamentos sexuais.

Uma avaliação negativa das meninas pesquisadas frente à gravidez na adolescência foi verificada em suas manifestações da idade ideal para ter um primeiro filho, a partir dos 20 anos. Mesmo levando-se em conta que respostas a questionários de pesquisas não refletem as práticas diante de assuntos que são permeados por aspectos de natureza moral (Batson \& Thompson, 2001) as colocações das meninas sugeriam possível sinceridade, visto preocupações manifestadas quanto ao sigilo da pesquisa. Nas meninas que afirmaram já terem tido relações sexuais (quase metade das investigadas), as idades indicadas para a primeira relação sexual partiam dos 15 anos, com maior concentração aos 16 anos. Algumas meninas não quiseram responder à questão que indagava sobre o início das relações sexuais, indicando que a possibilidade dessa ocorrência tenha sido maior do que o avaliado.

\section{Considerações finais}

Dentro das claras limitações do estudo realizado, os achados apontaram para possibilidades de que os jovens avaliados encontravam-se numa trajetória positiva de desenvolvimento em relação às dimensões verificadas, especialmente nos aspectos de competências sociais e atividades, e também quanto aos estados de desenvolvimento da identidade. No território que esses adolescentes habitavam havia escassez de recursos esportivos e de lazer, mas os serviços socioculturais oferecidos pelo equipamento público que eles frequentavam podem ter sido importantes fontes para absorção de recursos necessários ao bom desenvolvimento dessas condições. Fica a sugestão de novos estudos sobre desenvolvimento de jovens em condição de vulnerabilidade social de forma a contribuir para o desenvolvimento positivo dessa população.

\section{Referências}

Achenbach, T. M., \& Rescorla, L. A. (2010). Mental Health practitioners' guide for the Achenbach System of Empirically Based Assessment (ASEBA). (7a Ed). [Manual]. Burlington: University of Vermont.

Assis, S. G., Pesce, R. P., \& Avanci, J. Q. (2006). Resiliência. Enfatizando a proteção dos adolescentes. Porto Alegre: Artmed. 
Barreto, A. C. (2016). Paradigma sistêmico no desenvolvimento humano e familiar: a teoria bioecológica de Urie Bronfenbrenner. Psicologia em Revista, 22(2), 273-291. Recuperado de https://goo.gl/uEHPue

Batson, C. D., \& Thompson, E. R. (2001). Why don't moral people act morally? Motivational considerations. Current directions in psychological science, 10(2), 54-57. doi: 10.1111/1467-8721.00114

Bernardo, L. A. S., \& Oliveira-Monteiro, N. R. (2015). Problemas emocionais e de comportamento em adolescentes grávidas. Revista Psicologia Argumento, 33(81), 291-313. doi 10.7213/psicolargum.33.081.A006

Bronfrenbrenner, U. (2001). A Ecologia do Desenvolvimento Humano. Porto Alegre: Artmed.

Dias, A. C. G., \& Gomes, W. B. (1999). Conversas sobre sexualidade na família e gravidez na adolescência: a percepção dos pais. Estudos de Psicologia, 4(1), 79-71096, 1999. doi: 10.1590/S1413-294X1999000100006

Erikson, E. H. (1997). The life cycle completed. New York: W. W. Norton \& Company.

Kimmel, D. C., \& Weiner, I. B. (1998). La adolescencia: una transición del desarrollo. Espanha: Ariel.

Marcia, J. E. (1967). Ego identity status: relationship to change in self-esteem, "general maladjustment", and authoritarianism. Journal of Personality, 35, 118-123. doi: 10.1111/j.1467-6494.1967.tb01419.x

Matteson, D. R. (1972). Exploration and commitment: sex differences and methodological problems in the use of identity status categories. Journal of Youth and Adolescence, 6(4), 353-374. doi: 10.1007/BF02139239

Ministério da Saúde (2008). Pesquisa de conhecimentos, atitudes e práticas da população brasileira de 15 a 64 anos de idade (PCAP). Brasília: Autor. Recuperado de https://goo.gl/bQGUCN

Oliveira-Monteiro, N. R., Nascimento, J. O. G., Montesano, F. T., \& Aznar-Farias, M. (2013). Competência, problemas internalizantes e problemas externalizantes em quatro grupos de adolescentes. Psico-USF, 18(3), 427-436. doi: 10.1590/S1413-

82712013000300009

Oliveira-Monteiro, N. R., Freitas, J. V., \& Aznar-Farias, M. (2014). Gravidez: Associação de fatores de risco e proteção na adolescência. Revista Brasileira de Crescimento e Desenvolvimento Humano, 24(3), 354-360. doi: dx.doi.org/10.7322/jhdg.88977

Organização Mundial da Saúde [OMS] (2014). Health for the world's adolescents: A second chance in the second decade. Geneva, World Health Organization. Recuperado de http://apps.who.int/adolescent/second-decade/

Prado, J. L. (2008). Juventude cidadã: a prática da prefeitura de São Bernardo do Campo de políticas públicas para a juventude. São Paulo: Secretaria de estado da saúde de São Paulo. 
Reis, A. O. A., \& Oliveira-Monteiro, N. R. (2007). Sexualidade e procriação na ótica de jovens de periferias sociais e urbanas. Revista Brasileira de Crescimento e Desenvolvimento Humano, 17(2), 54-63. Recuperado de https://goo.gl/SYFD6W

Rocha, M. M. (2012). Autoavaliação de competências e problemas de comportamento entre adolescentes brasileiros: Um estudo de validação do Inventário de Autoavaliação para Jovens (YSR/2001). Tese de doutorado, Programa de Pós-Graduação em Psicologia Clínica, Instituto de Psicologia, Universidade de São Paulo, São Paulo, SP.

Ruotti, C., Massa, V. C., \& Peres, M. F. T. (2011). Vulnerabilidade e violência: Uma nova concepção de risco para o estudo dos homicídios de jovens. Interface - Comunicação, Saúde, Educação, 15(37), 377-89. doi: 10.1590/S1414-32832011005000004

Sandhu, D. (2015). Predictive factors of health-risk behaviours among male adolescentes. Pakistan Journal of Psychological Research, 30(1), 1-19.

Schoen-Ferreira, T. H. (2007). A adolescência e a formação da identidade: uma proposta de avaliação e intervenção. Tese de doutorado, Programa de Pós-graduação em Ciências Aplicadas à Pediatria,Universidade Federal de São Paulo, São Paulo.

Schoen-Ferreira, T. H., Meneghuelli, N. \& Aznar-Farias, M. (2005). Validação semântica do Extended Objective Measure of Ego Identity Status 2 - EOMEIS-2. In Instituto Brasileiro de Avaliação Psicológica (Eds.), Congresso Brasileiro de Avaliação Psicológica, Porto Alegre: Autor

Schwart, S. J., Zamboanga, B. L., Meca, A., \& Ritchie, R. A. (2012). Identity around the world: An overview. New Directions for Child and Adolescent Development, 2012(138), 1-18. doi: 10.1002/cad.20019

Sukiennik, P. B. \& Salle, E. (2002). Depressão. Em M. C. O. Costa \& R. P. de Souza (Orgs.), Adolescência (pp. 340-349). PortoAlegre: Artmed

Teixeira, A. M. F. B., Knauth, D. R., Jandyra, M. G. F., \& Leal, A. F. (2006). Adolescentes e uso de preservativos:as escolhas dos jovens de três capitais brasileiras na iniciação e na última relação sexual. Cadernos de Saúde Pública, 22(7), 1385-1396. doi: 10.1590/S0102311X2006000700004.

Tesouro, M., Palomanes, M., Bonachera, F., \& Marínez, L. (2013). Estudio sobre el desarrollo de la identidad em la adolescência. Tendencias Pedagógicas, 21, 211-224. Recuperado de https://goo.gl/L6Kc7z

Zacarés, J. J., Iborra, A., Tomás, J. M., \& Serra, E. (2009). El desarrollo de la identidad en la adolescencia y adultez emergente: Una comparación de la identidad global frente a la identidad en dominios específicos. Anales de Psicología, 25(2), 316-329.

doi:10.6018/87931

Recebido / Received: 10/11/2016

Aprovado / Approved: 21/12/2016 